\title{
What works in falls prevention in Asia: a systematic review and meta-analysis of randomized controlled trials
}

\author{
Keith D. Hill ${ }^{1 *}$, Plaiwan Suttanon², Sang-I Lin ${ }^{3}$, William W.N. Tsang ${ }^{4}$, Asmidawati Ashari, ${ }^{5,1}$,
}

Tengku Aizan Abd Hamid ${ }^{5}$, Kaela Farrier ${ }^{1}$ and Elissa Burton ${ }^{1}$

\begin{abstract}
Background: There is strong research evidence for falls prevention among older people in the community setting, although most is from Western countries. Differences between countries (eg sunlight exposure, diet, environment, exercise preferences) may influence the success of implementing falls prevention approaches in Asian countries that have been shown to be effective elsewhere in the world. The aim of this review is to evaluate the scope and effectiveness of falls prevention randomized controlled trials (RCTs) from the Asian region.

Method: RCTs investigating falls prevention interventions conducted in Asian countries from (i) the most recent (2012) Cochrane community setting falls prevention review, and (ii) subsequent published RCTs meeting the same criteria were identified, classified and grouped according to the ProFANE intervention classification. Characteristics of included trials were extracted from both the Cochrane review and original publications. Where $\geq 2$ studies investigated an intervention type in the Asian region, a meta-analysis was performed.

Results: Fifteen of 159 RCTs in the Cochrane review were conducted in the Asian region (9\%), and a further 11 recent RCTs conducted in Asia were identified (total 26 Asian studies: median 160 participants, mean age:75.1, female:71.9\%). Exercise (15 RCTs) and home assessment/modification $(n=2)$ were the only single interventions with $\geq 2$ RCTs. Intervention types with $\geq 1$ effective RCT in reducing fall outcomes were exercise (6 effective), home modification (1 effective), and medication (vitamin D) (1 effective). One multiple and one multifactorial intervention also had positive falls outcomes. Meta-analysis of exercise interventions identified significant benefit (number of fallers: Odds Ratio 0.43 [0.34,0.53]; number of falls: 0.35 [0.21,0.57]; and number of fallers injured: 0.50 [0.35,0.71]); but multifactorial interventions did not reach significance (number of fallers $\mathrm{OR}=0.57[0.23,1.44]$ ).

Conclusion: There is a small but growing research base of falls prevention RCTs from Asian countries, with exercise approaches being most researched and effective. For other interventions shown to be effective elsewhere, consideration of local issues is required to ensure that research and programs implemented in these countries are effective, and relevant to the local context, people, and health system. There is also a need for further high quality, appropriately powered falls prevention trials in Asian countries.
\end{abstract}

Keywords: Falls prevention, Effectiveness, Asia, Elderly, Community

\footnotetext{
* Correspondence: Keith.Hill@Curtin.edu.au

'School of Physiotherapy and Exercise Science, Curtin University, GPO Box

U1987, Perth, WA 6845, Australia

Full list of author information is available at the end of the article
} International License (http://creativecommons.org/licenses/by/4.0/), which permits unrestricted use, distribution, and reproduction in any medium, provided you give appropriate credit to the original author(s) and the source, provide a link to the Creative Commons license, and indicate if changes were made. The Creative Commons Public Domain Dedication waiver (http://creativecommons.org/publicdomain/zero/1.0/) applies to the data made available in this article, unless otherwise stated. 


\section{Background}

Falls are recognized as a major cause of death and growing burden of disease world-wide [1, 2]. There has been strong growth in the available research evidence from randomized controlled trials investigating interventions to reduce falls among older people living in the community, with the most recent (2012) Cochrane review reporting 159 studies with 79,193 participants [3]. However, data from several developed countries indicate that despite this high volume of quality research, that key national indicators such as rates of falls related hospitalizations continue to increase (for example, in Australia, trend data from 1999 to 2013 indicate an average 2\% increase in age-standardized fall related hospitalizations per year) [4-6]. Importantly, if rates of fall related hospitalizations remain stable or increase, then with aging populations this means substantial growth in actual case numbers of people being hospitalized due to falls (see Fig. 8.3 in [4]).

Population aging is occurring at differing rates between countries [7]. Japan is the "oldest" country in the world, with $26 \%$ of its population aged over 65 years [8]. Many developed countries already have more than $15 \%$ of their population aged greater than 65 years, [8] and are expected to exceed $20 \%$ by 2050 . In contrast, many developing countries in Asia such as Malaysia, Thailand, Indonesia, Cambodia, Vietnam and the Philippines have less than 10\% of their population aged more than 65 years $[9,10]$. However, Asia is home to $60 \%$ of the world's population, [8] and has the fastest aging population of any region in the world [11]. There is a need for a strong preventive approach to minimize the risk of falls and associated injuries as countries' aging populations grow.

The available research provides mixed evidence about the magnitude of the problem of falls between countries. While it is widely accepted from prospective, large scale representative samples in a number of Western countries that between 30 and $39 \%$ of the population aged greater than 65 will experience one or more falls in a 12 month period, [12-14] the proportion of older people reporting falls in the limited number of studies in Asian countries has generally been lower and considerably varied (14-34\%, median 18\%) [15-17]. Limitations to the design of most of these studies in Asian countries include utilizing retrospective data collection (recall of falls in the preceding 12 months), and lack of representative sampling. There may also be some cultural factors influencing whether or not a fall will be reported by an older person, [18] which may contribute to varying and under-reporting of falls outcomes.

Factors contributing to falls risk may also vary between countries and cultures $[18,19]$. Differences in intrinsic factors may include sunlight exposure, diet, stature, exercise patterns and preferences, and knowledge and attitudes towards ageing and falls prevention. For example, in a recent qualitative study of older people in Thailand, falls prevention was not noted as a perceived benefit of exercise, and some family and cultural values were considered to be potential barriers to older people becoming involved in exercise [20]. Another qualitative study in India reported that older people often considered falls were random events, and not considered a health priority [21]. A study in China reported fatalistic perceptions about falls being common among older people, low levels of knowledge about falls prevention interventions were evident, and falls were often hidden from family and doctors, and were not often discussed openly [22]. Differences in extrinsic factors also exist, including the home and outdoor environment, and footwear [17, 21, 23]. Type of housing and flooring surfaces vary substantially across Asia, and outdoor environments such as footpaths are often poorly maintained or non-existent. A study in China highlighted environmental factors such as adequate lighting on stairs and adequate step width as protective of falls (and therefore poor lighting and narrow step width as risk factors for falls) [17]. Additionally, there can be differences in health services and systems, and engagement of older people in these. Focus is often on treatment of acute health conditions, and less on preventative care.

The growing evidence highlighting different rates of falls and potential falls risk factors between countries raises the question of whether interventions that have been shown to be effective in one country may need some tailoring if being introduced into countries that have considerable diversity to the country where the intervention was shown to be effective. One recent scoping review has considered this issue in the context of falls prevention studies that have been conducted in South East Asian countries [23]. This review reported some unique aspects of socioeconomic, geographical and cultural differences of South East Asian countries, and identified limited quality research investigating falls prevention interventions in this region. However, the majority of the studies reported were non-randomized trials, studies were limited to only South East Asian countries, and there was no quality assessment of the included studies. The objective of our systematic review paper was to review the randomized controlled trial research evidence conducted among older people living in the community across the Asian region to identify (1) the type and number of falls prevention interventions shown to be effective in Asian populations; and (2) gaps for future research investigating falls prevention interventions in Asian countries.

\section{Methods}

The 2012 community setting falls prevention Cochrane review was used as the basis for this review as it is the most recent and extensive systematic review and meta-analysis available on this topic [3]. Studies in the Cochrane review were categorized as to whether the study was undertaken in an (i) Asian, or (ii) non-Asian country. Additionally, a systematic review of the falls prevention literature published 
since the 2012 Cochrane review was also conducted, to identify more recent falls prevention RCTs conducted in the Asian region. This update component was guided by the Additional file 1: PRISMA checklist to ensure the results are reported systematically [24].

Intervention types were classified according to the Prevention of Falls Network Europe (ProFaNE) classification as (i) single interventions, (ii) multiple interventions (two or more single interventions, applied to all participants), and (iii) multifactorial interventions (two or more interventions, targeted to an individual's risk factor profile, often based on a falls risk assessment process - different participants receive a different mix of interventions) [25]. Single interventions were further classified as described in the ProFANE classification as (a) exercise, (b) medication, (c) psychological, (d) environmental/assistive technology, (e) interventions to increase participant knowledge, (f) surgical interventions (eg cataract surgery, cardiac pacemaker surgery), (g) interventions to address incontinence, and (h) fluid or nutritional therapy. This intervention classification system was used in the 2012 Cochrane review, [3] and was also used to classify the more recent RCTs.

\section{Eligibility criteria for RCTs published since 2012 Cochrane review}

The additional studies included in this review met the following eligibility criteria: peer reviewed articles published from January 2012 - November 2016; studies undertaken in Asia; randomized controlled trials; people aged 60 years and over - at least $50 \%$ of the sample; living in the community, and reporting at least one falls outcome.

\section{Information sources for additional RCTs}

Studies were identified from six databases: Medline (Proquest), CINAHL, PubMed, PsycINFO, SPORTDiscus and Scopus for the time period described above. Reference lists from the included articles were also scanned. Only papers in English were included, no unpublished data, conference proceedings, books, poster abstracts or theses were included.

\section{Search strategy for additional RCTs}

The search strategy used a mix of keywords which could be identified in the title and/or abstract. The search strategy included fall* [Title/abstract] AND communit* [Title/abstract] AND RCT [Title/abstract] OR randomi* controlled trial [Title/abstract]. There were differences between the databases for language and syntax, and where this occurred only the abstract was searched.

\section{Study selection for additional RCTs}

Study selection was a three-stage process, stage one involved one author (KF) screening all of the additional identified papers based on their titles; stage two involved screening abstracts according to the eligibility criteria; and stage three involved two independent researchers (KF and EB) screening full articles. Where disagreements occurred the two researchers discussed the reasons for their decision, referring back to the eligibility criteria throughout the process, until they reached consensus.

\section{Data collection process}

Characteristics of the intervention programs undertaken in Asian countries were retrieved from original publications independently by two of the researchers ( $\mathrm{KH}$ and $\mathrm{EB} / \mathrm{KF}$ ). Comparing characteristics of the interventions (eg for exercise - type, duration, frequency) provided an opportunity to explore factors that may have contributed to the success or failure of different interventions in Asian populations.

\section{Study quality assessment for additional RCTS (post 2012 Cochrane review)}

The Cochrane Collaboration's risk of bias tool [26] was used by two researchers (KF and $\mathrm{EB}$ ) independently to assess methodological quality of each paper (study quality of the Cochrane review papers can be accessed in the Cochrane review). Categories assessed by the tool include sequence generation, allocation concealment, blinding (staff, participants and outcome assessors), incomplete outcome data, selective outcome reporting and other sources of bias [26]. Risk of bias was assessed as "low risk", "medium risk" or "high risk" [26]. Where disagreement occurred between the two assessors a third assessor (EJB) also assessed those domains, discussed with the two independent assessors their outcomes and came to a consensus.

\section{Analysis}

Extracted data from all the Asian studies were tabulated, and overall sample demographics and characteristics of the interventions undertaken in Asian countries summarized, to assist in interpretation of the primary outcomes.

The primary analyses for this paper involved meta-analysis of falls outcomes (e.g. number of falls) by intervention type (according to the ProFaNE classification) where there were two or more randomized controlled trials conducted in Asian countries with comparable data.

For the meta-analyses, number of participants and events (falls, fallers, non-injurious falls) for each group were sourced from all original articles (including Cochrane review articles). RevMan 5.3 software was used to conduct the analyses and generate the forest plots, using a Mantel-Haenszel's fixed effect model (with odds ratios and 95\% confidence intervals calculated) [27]. Visual inspection of the forest plots and the $\mathrm{I}^{2}$ statistic were used to assess heterogeneity. When heterogeneity was deemed as high $\left(\mathrm{I}^{2}>50 \%\right)$ a random effects model was applied. Where a study had two intervention groups and one control group, the intervention groups (i.e. 
dichotomous data) were summed (as recommended by Cochrane) for both the outcome (e.g. number of falls) and the sample size independently [27]. Subgroup analysis was undertaken where two or more programs/services within the intervention type could be grouped, for example Tai Chi within exercise interventions. Studies with group differences in baseline characteristics were omitted. Statistical significance was considered at $p \leq 0.05$. Some studies reported more than one single intervention arm compared to a control arm. In these cases, if separate falls data were reported for each of the single interventions, then these were reported separately in the tables and meta-analyses.

\section{Results}

Of the 159 randomized controlled trials reported in the 2012 Cochrane review [3], 15 (9\%) were conducted in Asian countries (Japan, $n=6$; [28-33] Taiwan $n=6$; [34-39] Thailand, $n=2$; [40, 41] and China/Hong Kong, $n=1$ [42]). The remaining 144 published randomized trials were from non-Asian countries, including the United States of America $(n=34,21 \%)$, Australia $(n=27$, $17 \%)$, the United Kingdom $(n=27,17 \%)$, Canada $(n=12$, $8 \%)$, the Netherlands $(n=9,6 \%)$, New Zealand $(n=6,4 \%)$, Germany $(n=6,4 \%)$, and other parts of Europe $(n=16$, $11 \%)$. Several other studies were conducted across two or more countries $(n=4)$, although none of these included countries in Asia. Table 1 reports the number of randomized trial interventions classified by ProFaNE classification type, between Asian and non-Asian populations.

An additional 11 RCTs were identified that were conducted in Asian populations, met the inclusion criteria for the community based falls prevention Cochrane review, and were published after the 2012 Cochrane review (Japan $n=6$; [43-48] Taiwan $n=2$; $[49,50]$ and China/Hong Kong, [51] Malaysia [52] and Singapore [53] $n=1$ each). See Fig. 1 for study selection flowchart to identify papers after 2012. Seven of the additional studies published since the 2012 review were exercise interventions.

Combining the Asian studies in the 2012 Cochrane review and subsequent Asian RCTS, the only single intervention types that had more than one randomized controlled trial conducted in Asia were exercise $(n=15)$, and environment (home hazard assessment and modification, $n=2[38,48])$. There were also five multiple intervention studies [34, 37, 41, 50, 53] (two with two or more single intervention arms included as separate single interventions in the single intervention analyses $[34,37])$ and four multifactorial intervention studies conducted in Asia $[35,36,39,40]$.

\section{Samples}

Overall, the sample size for the studies in Asia included in the 2012 Cochrane review were relatively small (median 150, minimum 52, maximum 1043) (Table 2), whereas for the full Cochrane review [3], the median sample size across all randomized trials irrespective of where the trial was conducted was 230 (minimum 10, maximum 9940). The more recent Asian randomised controlled trials (published since the Cochrane review) have been substantially larger than those pre-2012 (median sample size 196, minimum 68, maximum 710). The samples were mixed in terms of the age inclusion criteria, with two studies including people aged over 50 years [28, 52], five had samples aged $\geq 60$ years [37, 39, 41, 49, 51], 14 had samples aged $\geq 65$ years $[29,32-36,38,42,44,45,47,48,50,53]$, three samples were aged $\geq 70$ years $[30,40,46]$, one sample was aged $\geq 73$ years [31], and one sample was aged $\geq 75$ years [43]. The average age of participants across the included studies in Asia was 75.1 years, and samples were on average $71.9 \%$ female (five studies had female only samples [29-31, 46, 51]).

\section{Intervention types}

Table 1 reports details of individual intervention types conducted in Asia relative to the rest of the world, while Table 2 summarizes the main characteristics of the trials conducted in Asia, grouped by intervention type, including details of whether or not the intervention was effective in reducing the rate of falls or number of people falling.

\section{Single interventions \\ Exercise interventions}

Fifteen trials from Asia reported results for an exercise intervention as a single intervention, with only six reporting one or more positive fall related outcomes $[31,42-44,46,49]$. The exercise trials generally had small sample sizes $(60 \% n<70$, median sample size $n=105)$. Three of the effective exercise programs used Tai Chi $(20 \%$ of all exercise trials) either as the sole intervention [42, 49] or combined with other balance and strength exercises [31]. In contrast, only five of the $51(10 \%)$ exercise trials from the 2012 Cochrane review conducted outside of Asia involved Tai Chi. The other effective exercise approaches in Asian studies used an obstacle course [43], a multi-target stepping program [44], and a group balance and strength training program [46]. Only one of the ineffective exercise trials used Tai Chi, [34] other ineffective programs used home programs, $[29,38,52]$ group programs (balance [47] or multimodal [45]), a group exercise program combined with trail walking, [33] combined group and home based program, [28] and a square stepping program [32]. Effective exercise programs ranged from 24 to 52 weeks duration, and had from 1 to 8 times per week of recommended exercise (included both supervised and home-based sessions).

In a separate review, Sherrington and colleagues reviewed 54 randomized controlled trials evaluating exercise interventions to reduce falls, and identified several key criteria that appeared to differentiate effective from ineffective falls prevention trials [54]. Two of the key 


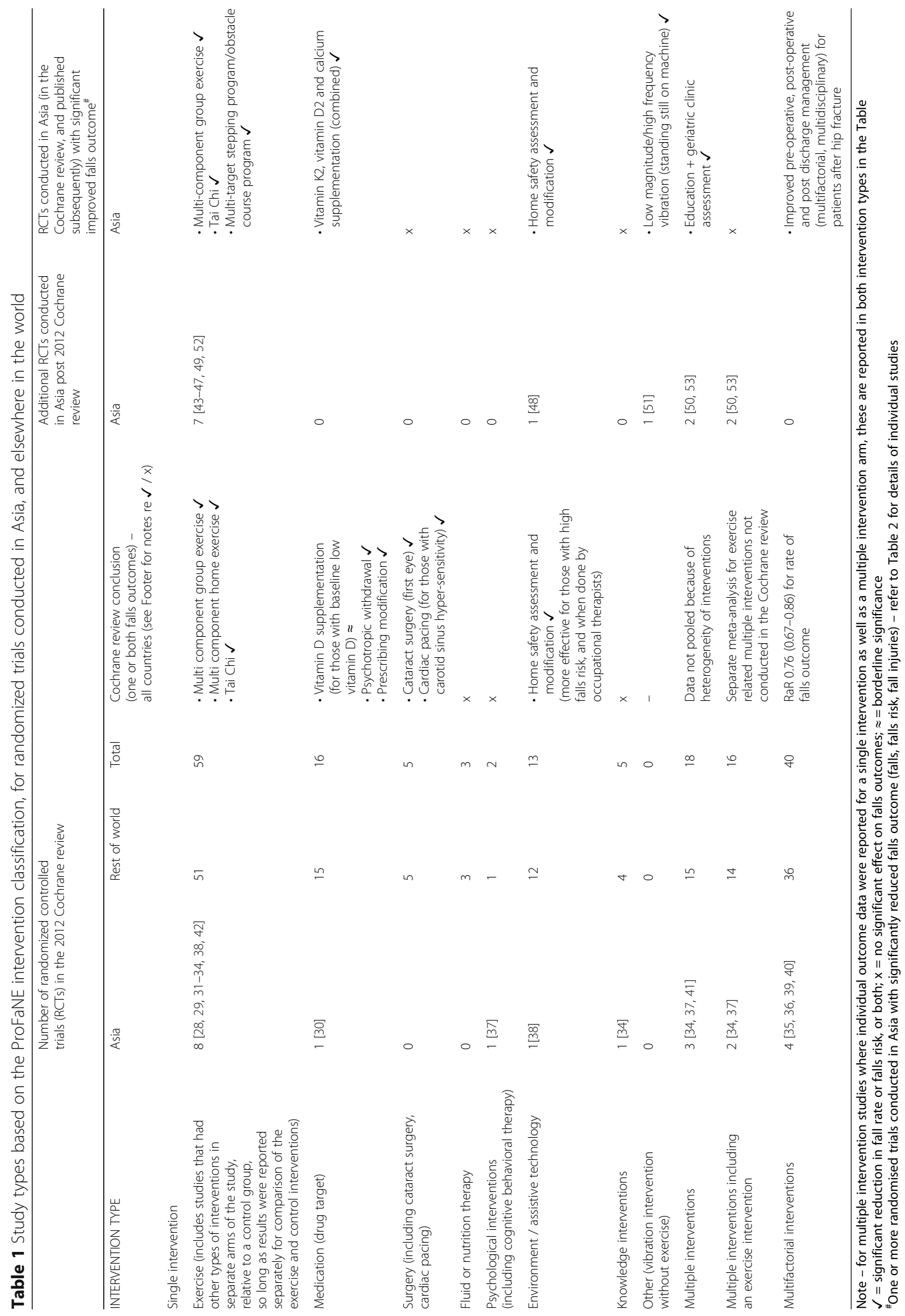




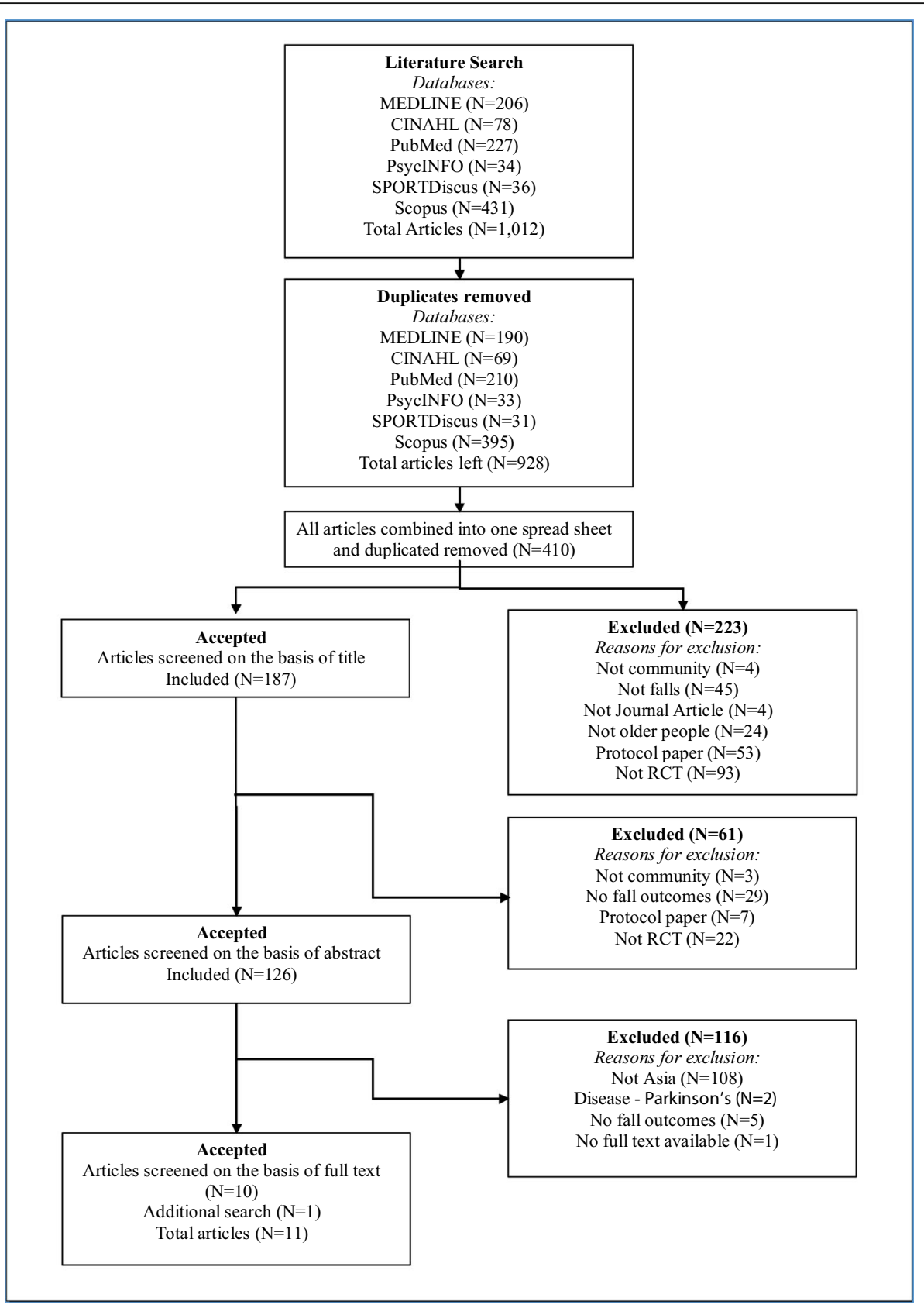

Fig. 1 Study selection flowchart for randomised trials published after the 2012 Cochrane

criteria related to: (1) the exercise intervention having a moderate to high challenge to balance; and (2) a minimum of $50 \mathrm{~h}$ overall exercise dosage. Of the six effective exercise trials in Asia, four (67\%) met both of these criteria $[31,42,46,49]$. The other two effective exercise studies $[43,44]$ met the "challenge to balance" criteria, but involved substantially less than the $50 \mathrm{~h}$ duration. Three of the ineffective exercise studies met both of Sherrington's criteria, but were likely to be underpowered (sample sizes of 57 [29], 68 [52] and 93 [47]). Most of the remaining ineffective exercise interventions did not meet the dosage criteria, although the majority met the balance challenge criteria. Although adherence to the exercise programs was reported in ten of the studies, the method of reporting was variable, making comparisons difficult. The study by Huang and colleagues reported high attrition in the Tai Chi group (52\%) over the five month intervention [34]. All except 


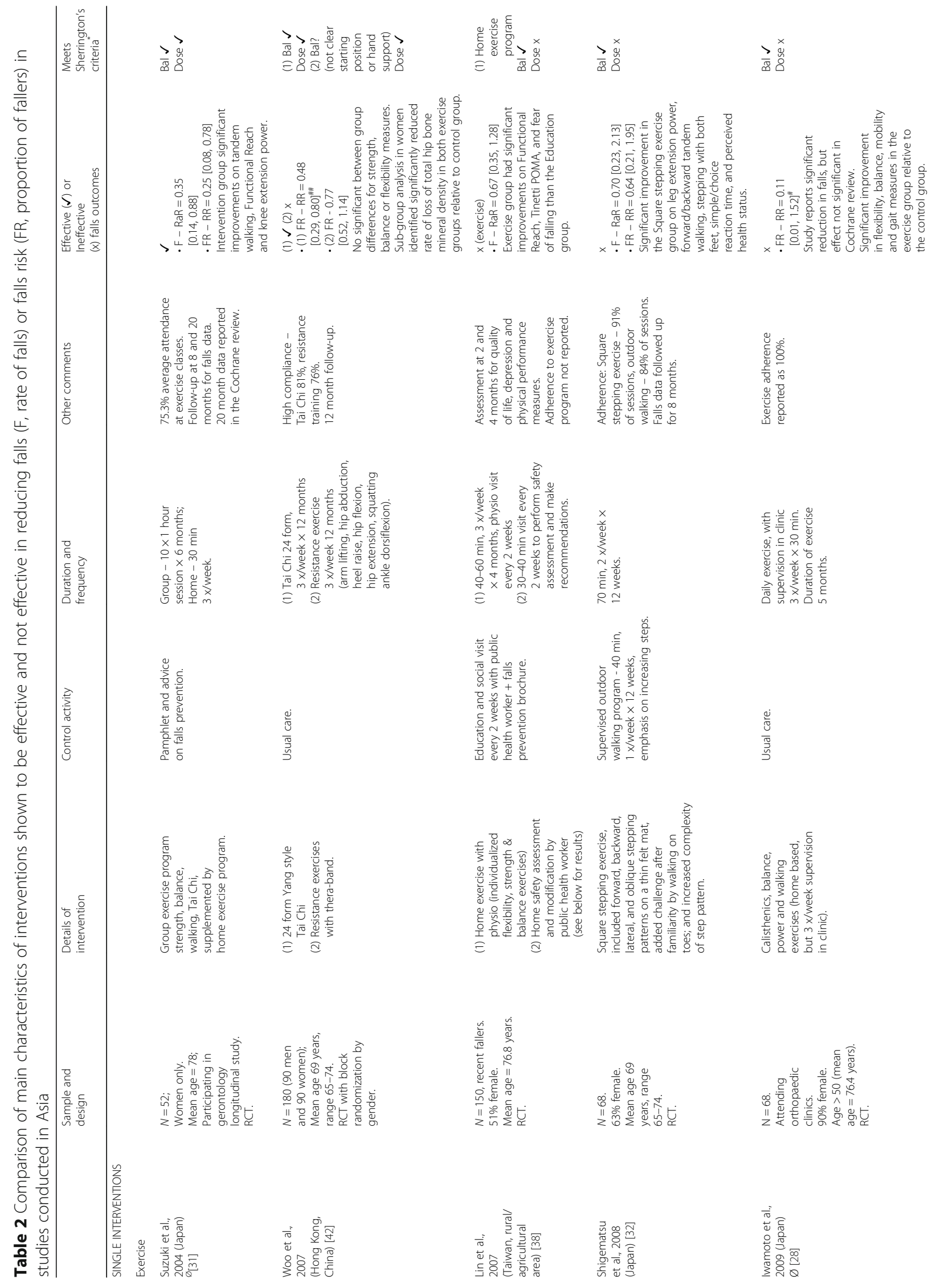




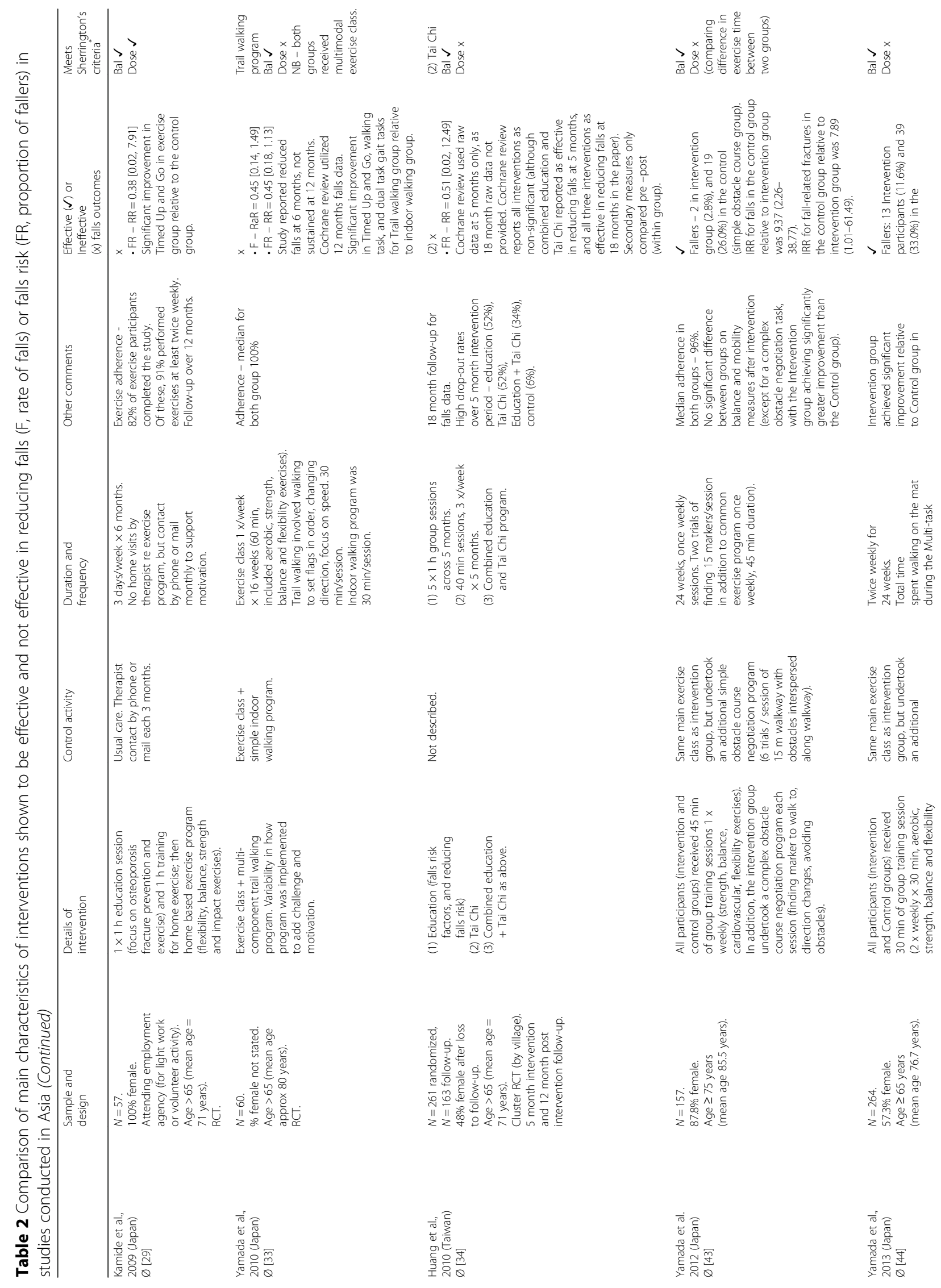




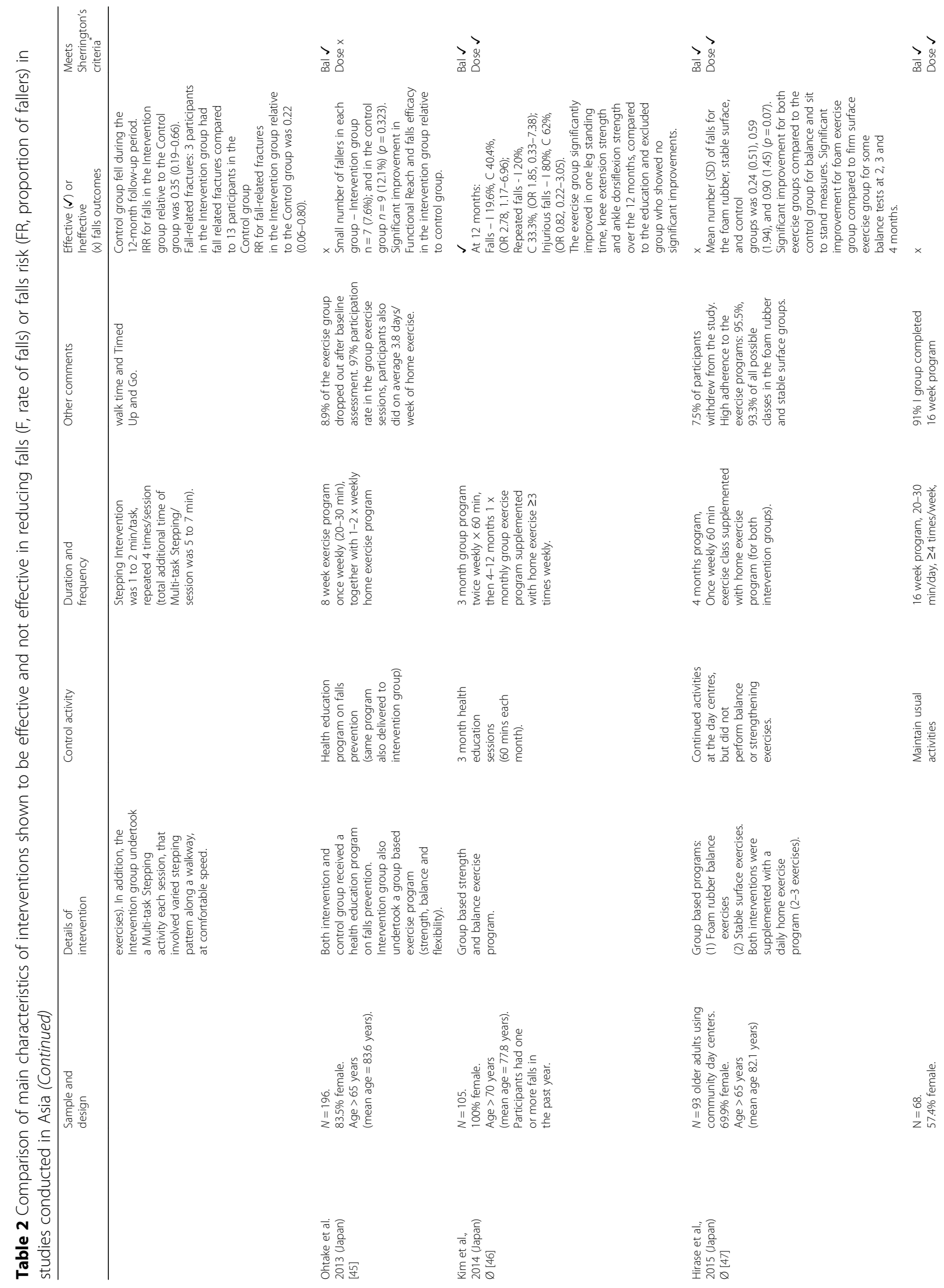




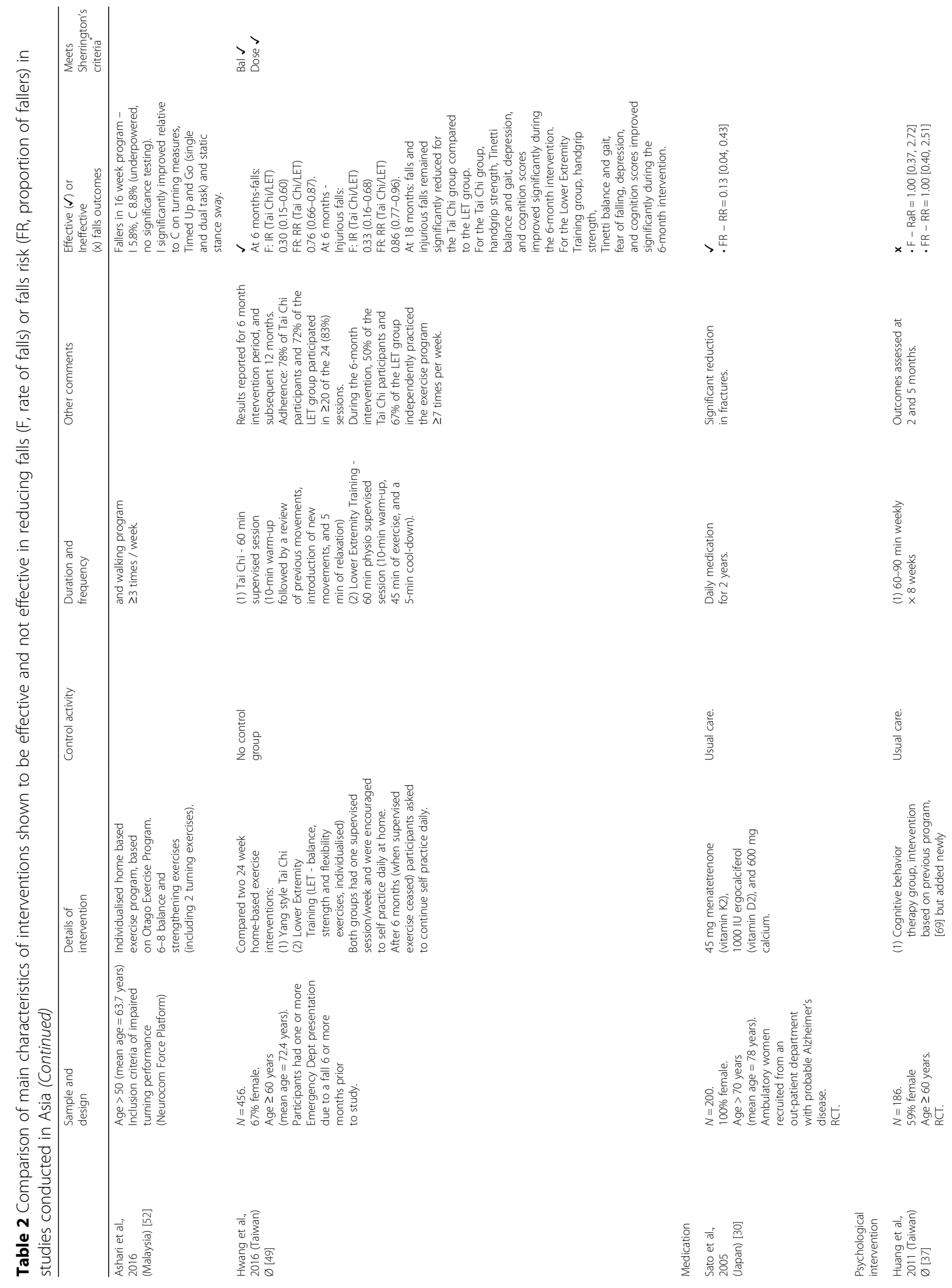




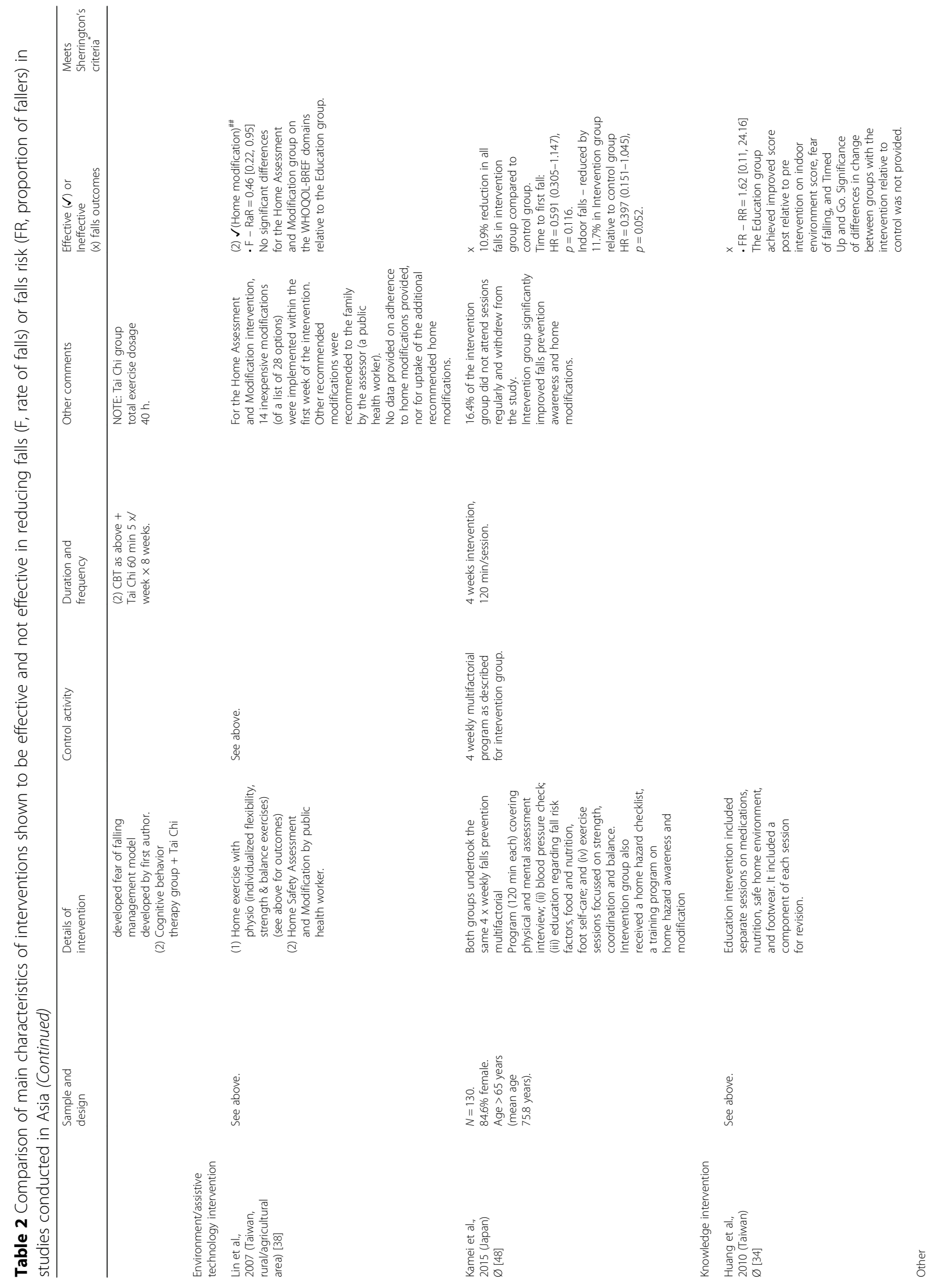




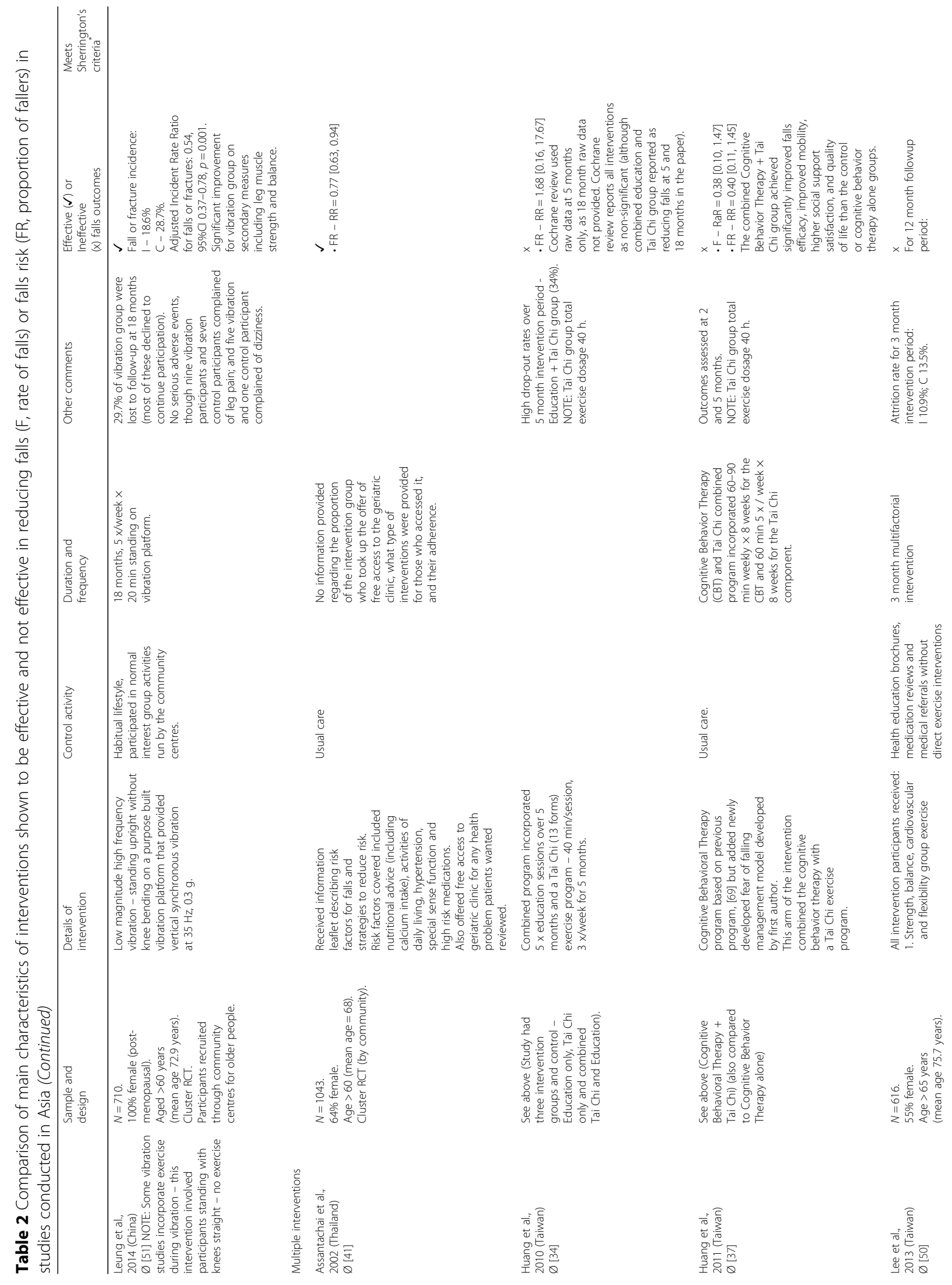




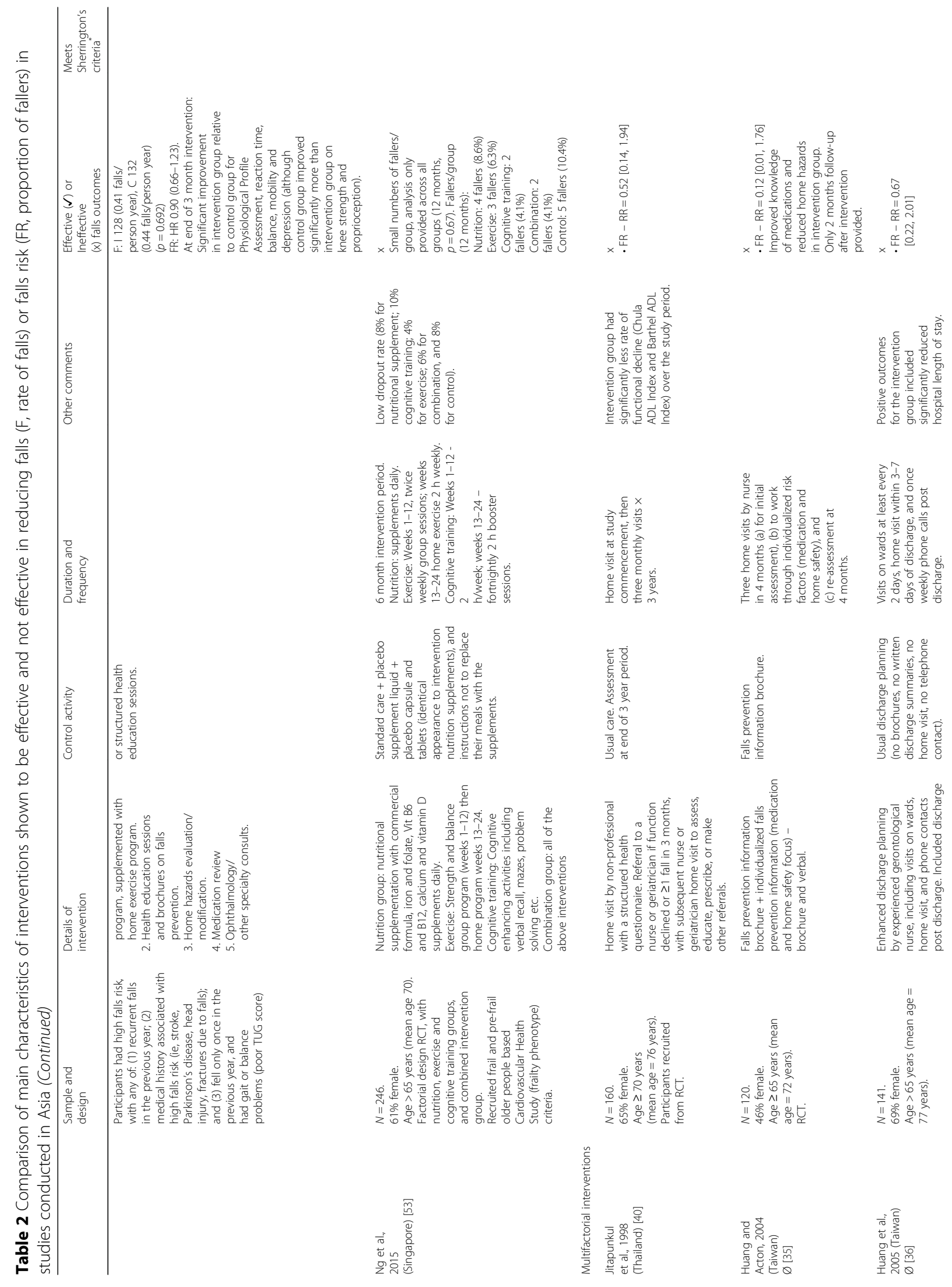


Hill et al. BMC Geriatrics (2018) 18:3

Page 14 of 21

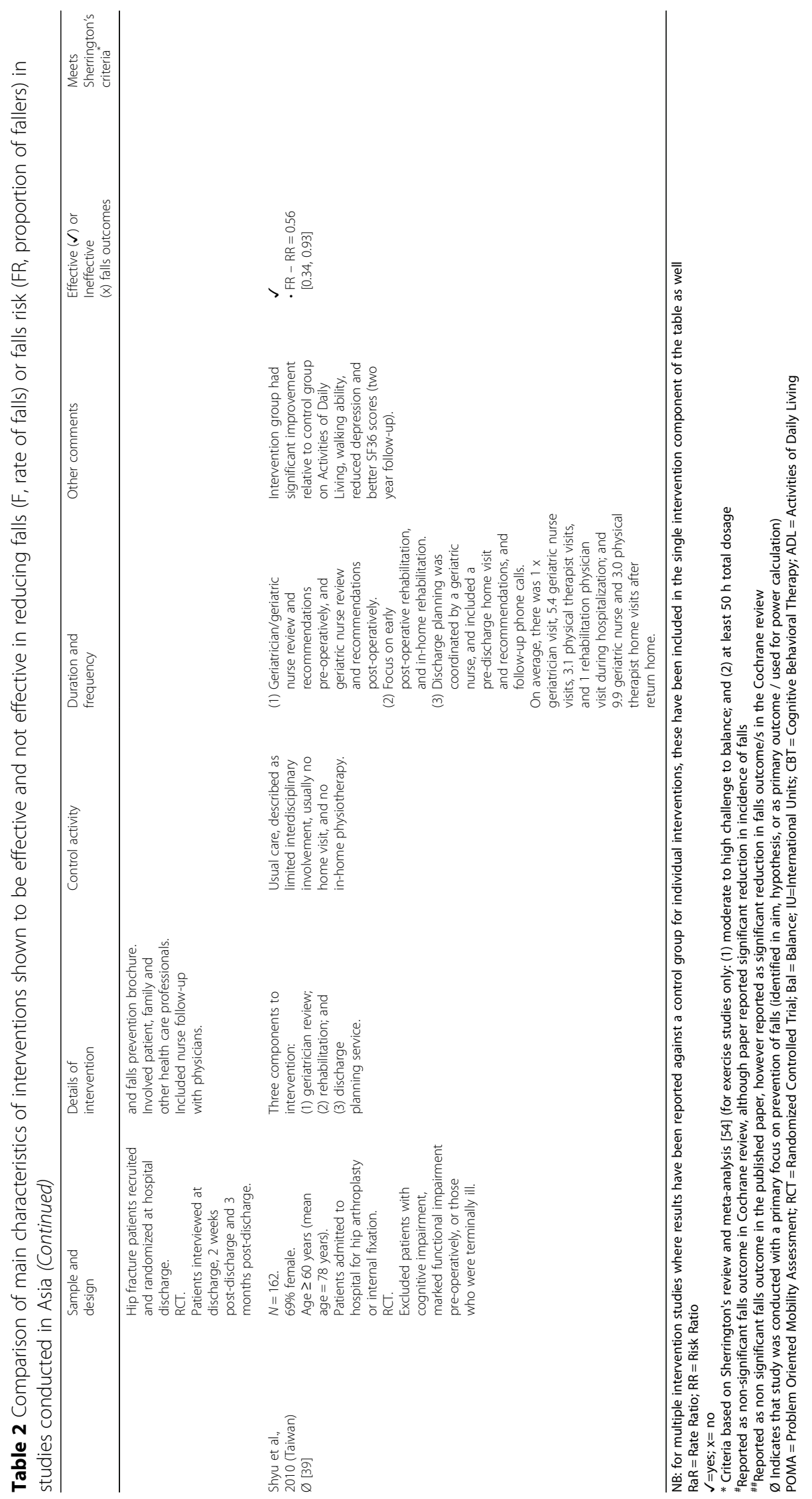


two of the studies reported significant improvements in the secondary balance, strength, or mobility related measures in the exercise intervention relative to the control group [34, 42].

\section{Medication interventions}

Medication interventions can include approaches to review or reduce medications overall, or specifically target high risk medications such as psychotropic medications; or provide supplementation to improve fall related outcomes [3]. Only one Asian study investigated a medication related intervention, by evaluating the effect of two years supplementation with vitamin $\mathrm{K} 2$, vitamin D2 and calcium, compared to usual care, in older women with probable Alzheimer's disease [30]. There was no effect on falls outcomes, but a significant reduction in fractures in the medication intervention group.

\section{Psychological interventions}

One study investigated the effectiveness of Cognitive Behavioral Therapy (CBT) alone, and in combination with Tai Chi, relative to a control group receiving usual care [37]. Neither CBT alone or combined with Tai Chi reduced falls outcomes. CBT alone did not result in significant improvements in any secondary measures, including falls efficacy, relative to the control group (see multiple interventions for outcomes for the combined Tai Chi and CBT intervention group).

\section{Environment/assistive technology interventions}

Two studies investigated home assessment and modification interventions. In a Japanese study, Kamei et al. evaluated the effect of a home hazard checklist and a training program on home hazard awareness for older people, superimposed on a multifactorial assessment and intervention program received by both the intervention and control group (both groups received a falls risk factor education program, exercise, blood pressure review, and physical and cognitive assessments) [48]. Although the intervention group significantly increased their falls prevention awareness and home modifications implemented, there was no significant reduction in falls. Another randomized trial in Taiwan compared a home assessment and modification intervention against an education intervention, and an exercise intervention [38]. The home modification program was conducted by a public health worker, and involved a standard assessment, provision of 14 standard, inexpensive modifications (eg removal of loose mats, marking of step edges, rectification of poor lighting), and recommendations regarding another 14 modifications if required. Although the home safety assessment and modification intervention was reported by Lin et al. [38] as not achieving a significant reduction in rate of falls, the reduction was significant in the Cochrane review analysis [3]. There was no information provided about the range of additional recommendations made, nor the level of adherence with the implemented or recommended home modifications.

\section{Knowledge interventions}

One randomized trial from Taiwan investigated the effect of knowledge based interventions provided to older people on reducing risk of falls [34]. The education intervention involved five one hour group education sessions over five months, targeting separate important risk factors at each session [34]. While the intervention achieved improved knowledge about falls risk, there was no reduction in falls outcomes.

\section{Other single interventions}

There were a number of additional interventions that have been shown to be effective in reducing falls, falls risk or falls injuries in the 2012 falls prevention in the community setting Cochrane review, which have not been investigated in any randomized controlled trials in Asian countries [3]. These include (a) medication prescription review; and high-risk medication withdrawal (eg psychotropic medications); (b) cataract surgery (first eye); (c) changing from bifocal or multifocal glasses to distance glasses for outdoors mobility; (d) cardiac pacing surgery (for carotid artery hypersensitivity); and (e) footwear (anti-slip shoe device for icy conditions). Vitamin D supplementation, which was considered effective in reducing falls in at-risk populations by the Cochrane review, has also not been evaluated for its effect on falls in Asian populations as a single vitamin supplement (Sato and colleagues evaluated supplementation of vitamin $\mathrm{K} 2$, vitamin D2 and calcium as a single intervention) [30]. Some of these interventions may be inappropriate in many parts of Asia (eg anti-slip shoe device for icy conditions). However, the other intervention types are likely to have direct or perhaps modified applicability for older people in Asia.

One recent trial in Asia has been classified as "Other" under the single intervention studies - an 18 month investigation of low-magnitude high-frequency vibration program in post-menopausal women in China/Hong Kong $(n=710)$ [51]. Vibration interventions often utilize exercises while performing vibration, [55] and so may be classified under exercise interventions, however the study by Leung and colleagues had participants standing with straight knees on the vibrating platform for the duration of the vibration procedure $(20 \mathrm{~min} /$ session). The group receiving the vibration therapy had significantly reduced Hazard Ratio for the 
combined outcome of "falls or fractures" ([0.56, $0.40-$ $0.78, p=0.001)$.

\section{Multiple interventions}

Five randomized controlled trials evaluated the effect of a multiple intervention approach to reducing falls $[34,37,41,50,53]$. Only one of the trials was effective, providing education (brochure targeting a number of important falls risk factors) and free access to a geriatric clinic as required for the intervention group [41]. Although effective, no details were provided about the uptake, type of interventions, or adherence to recommended interventions for the intervention group. The ineffective interventions included a combined Tai Chi exercise program and education program (each of these two components were also evaluated in isolation); [34] a combined Tai Chi and Cognitive Behavioral Therapy program; [37] a strength, balance, and fitness exercise program combined with health education, home assessment and modification, medication review, and ophthalmology or other specialty consultations; [50] and a factorial design study with participants receiving one or more of nutrition, exercise, and cognitive training interventions [53]. Tai Chi was shown to be an effective exercise intervention to reduce falls when used as a single intervention in three Asian studies, [31, 42, 49] and in non-Asian countries [3]. In both of the ineffective multiple intervention studies incorporating Tai Chi, the Tai Chi component did not incorporate the $50 \mathrm{~h}$ of exercise recommended to improve likelihood of achieving a significant reduction in falls [54].

\section{Multifactorial interventions}

Four of the randomized controlled trials conducted in Asia utilized a multifactorial falls prevention intervention [35, 36, 39, 40]. Two of these targeted a high-risk population (patients with hip fracture returning home after surgery), and utilized improved discharge planning and post discharge follow-up, including home visit/s [36, 39]. The study by Shyu and colleagues also incorporated a strong interdisciplinary care model pre and post-surgery [39]. The trial conducted by Jitapunkul and colleagues utilized a regular (three monthly) home visit health screening process by a non-health professional, with subsequent referral to a nurse or geriatrician if there was recent functional decline or $\geq 1$ fall in the preceding three months. Similarly, the study by Huang and Acton utilized a targeted falls risk brochure, followed up by targeted risk factor management support by a visiting nurse (focussing on medication and home safety) [35]. Only the trial by Shyu and colleagues was effective in reducing falls in the intervention group [39].

\section{Quality of the studies}

Risk of bias was reported for all studies published in the 2012 Cochrane review. The Cochrane Collaboration's tool for assessing the risk of bias was used to evaluate quality of the RCTs published since Gillespie et al.'s Cochrane review. Most of the recent studies had low to medium risk of bias (Table 3). Two studies had low risk of bias across all domains, $[49,52]$ with all the other studies having at least one section that was unclear (authors did not provide enough evidence). The only high risk of bias was for sequence generation and blinding in the study by Ohtake et al. [45] because participants were categorized by the day of the week and they did not blind the participants, personnel or outcome assessors. The risk of bias for the studies was viewed as low to medium due to sections of data in a number of the categories not being available, and are known to be essential for conducting high quality RCTs (blinding, allocation concealment).

\section{Meta-analyses}

For intervention types with two or more studies from the Asian region with comparable data available, a metaanalysis was conducted. Only the exercise $(n=15)$ intervention of the single interventions, and the multifactorial interventions $(n=4)$ were included in the meta-analyses (Fig. 2). The Ashari et al. study [52] was not included in the exercise meta-analysis due to significant differences between the groups at baseline. Woo and colleagues [42] had two intervention groups and therefore dichotomous (outcome and sample) data for the intervention groups only were combined, as described in the methods. Results from the meta-analysis indicate that exercise achieved significant reduction in number of fallers (OR: 0.43 [0.34-0.53]), number of falls (OR: 0.35 [0.21-0.57]) and number of fallers injured (OR: 0.50 [0.35-0.71]). Heterogeneity was at appropriate levels for the exercise intervention meta-analyses.

A separate subgroup meta-analysis was conducted for the Tai Chi exercise interventions. Results indicated Tai Chi achieved significant reduction in the number of falls (OR: $0.24[0.13-0.47]$ ) and number of fallers (OR: 0.46 $[0.30-0.70])$, although it must be noted that there was high heterogeneity for the number of fallers $\left(\mathrm{I}^{2}=67 \%\right)$ and therefore these results should be used with caution. Separate subgroup analysis was not able to be undertaken for the two home exercise program studies due to the Ashari and colleagues study [52] having differences at baseline discussed previously. No other sub-group analyses were possible.

The meta-analysis of the multifactorial interventions did not reach significance for number of fallers (OR: $0.57[0.23-1.44])$. Although there were two home assessment and medication trials, they reported different falls 
Table 3 Quality of the studies

\begin{tabular}{|c|c|c|c|c|c|c|}
\hline Study & $\begin{array}{l}\text { Sequence } \\
\text { generation }\end{array}$ & Allocation concealment & Blinding & Incomplete outcome data & Selective outcome reporting & Free of other bias \\
\hline Ashari et al. 2016 & + & + & + & + & + & + \\
\hline Hirase et al. 2015 & $?$ & + & $?$ & + & + & $?$ \\
\hline Hwang et al. 2016 & + & + & + & + & + & + \\
\hline Kamai et al. 2015 & $?$ & $?$ & $?$ & + & + & $?$ \\
\hline Kim et al. 2014 & + & $?$ & + & + & + & $?$ \\
\hline Lee et al. 2013 & + & + & $?$ & + & + & $?$ \\
\hline Leung et al. 2014 & + & + & + & + & + & $?$ \\
\hline Ng et al. 2015 & + & + & + & + & + & $?$ \\
\hline Ohtake et al. 2013 & - & $?$ & - & $?$ & + & $?$ \\
\hline Yamada et al. 2012 & + & + & $?$ & $?$ & + & $?$ \\
\hline Yamada et al. 2013 & $?$ & $?$ & + & $?$ & + & $?$ \\
\hline
\end{tabular}

Note. Bias was scored as low risk (+), or high risk (-) or unclear (?). [26]

outcome data, so meta-analysis was not able to be performed. Similarly, for the falls risk outcome for the multifactorial intervention classification, there were not two studies reporting this outcome to allow metaanalysis. Similar to the Cochrane review, meta-analysis of the multiple interventions was not possible because of the diversity of the intervention types combined as multiple interventions.

\section{Discussion}

Falls among older people remain a major public health problem world-wide. Substantial inroads are being made, with growing research evidence of single, multiple and multifactorial interventions being effective in reducing falls. However, the results of this focussed review of the subgroup of falls prevention randomized trials for the community setting conducted in countries in Asia, a

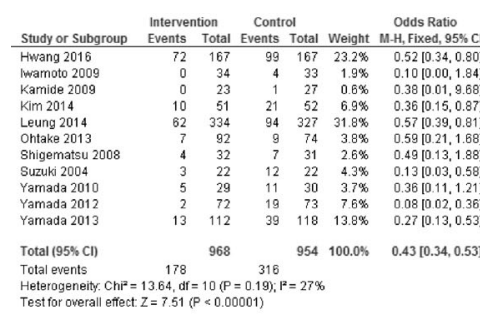

Tetion

\section{b}

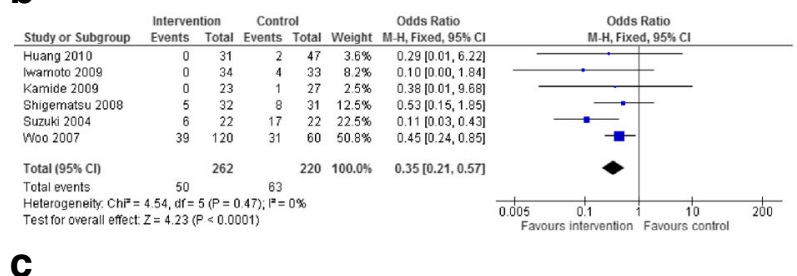

C

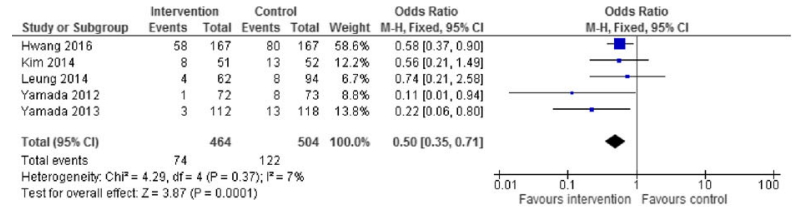

d

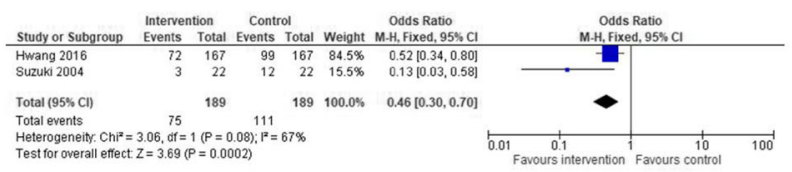

e

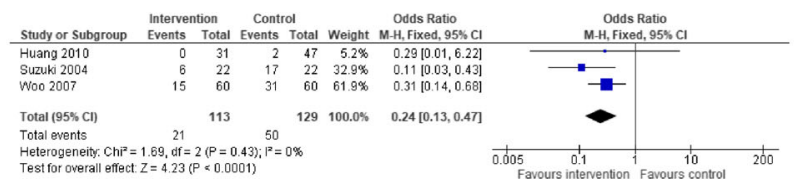

f

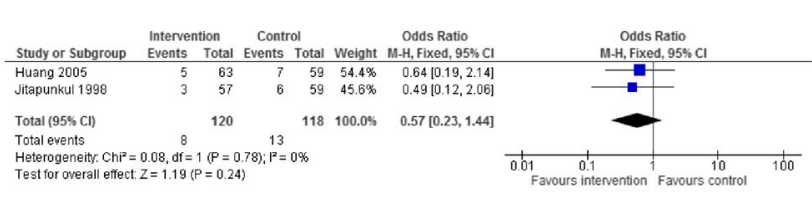

Fig. 2 Meta-analyses of intervention types that included two or more interventions from the Asian region. a. Number of fallers - Exercise, b. Number of falls - Exercise, $\mathbf{c}$. Number of fallers injured - exercise, d. Number of fallers - exercise using Tai Chi, e. Number of falls - exercise using Tai Chi, f. Number of fallers - Multifactorial Interventions 
where $60 \%$ of the world's population live, indicates substantial gaps. Not only has there been limited falls prevention research conducted in Asian countries, but where studies have been conducted, sample sizes are generally small, and only 11 of the 30 interventions evaluated in the 26 studies in Asia achieved a significant reduction in one or more falls outcomes $(37 \%)$. The meta-analysis results where more than one study was able to be pooled for an intervention type (exercise and multifactorial interventions) indicated that only exercise was effective in reducing falls in Asian populations.

Exercise is by far the most researched intervention type in Asian countries (as it is world-wide), with over half of the studies reported having an exercise component. Not surprisingly, Tai Chi was commonly investigated, and was effective in reducing falls outcomes in three studies in Asian countries, and in the sub-group meta-analysis. Although Tai Chi has been shown to be acceptable [56] and to reduce falls-related outcomes in non-Asian countries, [3] it does seem to be a preferred exercise approach for investigation and implementation in Asian countries. Adherence to the successful Tai Chi programs conducted in Asia was high (75-81\%), though similarly high adherence to Tai Chi has been reported in studies conducted in other countries $(68-80 \%)$ [57, 58]. Of note though, nine of the 15 studies investigating exercise in Asian countries were not effective, and the majority of these did not meet the criteria recommended by Sherrington [54] to be likely to be effective in reducing falls (having a moderate challenge to balance, and minimum of $50 \mathrm{~h}$ exercise dosage). Three studies that did meet these criteria were not effective, but were substantially underpowered to identify a reduction in falls related outcomes [29, 47, 52]. Other exercise approaches that were effective in single studies were an obstacle course, [43] a multi-target stepping program, [44] and a group balance and strength training program [46].

In an updated meta-analysis by Sherrington published around the time of this paper's publication, more stringent dosage criteria (greater than three hours per week) were identified as achieving the greatest benefit in reducing falls (23\% reduction) [59]. Higher exercise dosages, and sustained exercise (lifelong behaviour change) are clearly more desirable. However, low levels of sustained participation have been reported in falls prevention exercise programs, [60] suggesting that strategies such as starting off with lower dosages and gradually building up, and embedding behaviour change elements into exercise programs to support sustained and increased participation may be required to achieve this high dosage. Future exercise studies should aim to adopt a method that meets the updated Sherrington criteria while being culturally relevant, as well as being adequately powered for falls outcomes.
These results have substantial implications for falls prevention research and practice in Asia. It is often assumed that interventions shown to be effective in randomized trials or meta-analyses in one country will be generalizable elsewhere. However, researchers in the falls prevention area, $[23,61]$ and in other areas of health (eg hepatocellular carcinoma) [62] have called for local research in Asian and or developing countries to address key gaps and differences. In this context, recent research has also highlighted the diverse range of factors that may complicate or reduce likely effectiveness of directly translating falls prevention approaches found to be effective in nonAsian countries into Asian countries [18, 19, 23]. Some of these differing factors include: (1) role of family (including filial piety); (2) indoor (including floor surfaces) and outdoor environments; (3) regularly worn footwear in many parts of Asia differ from what is considered the ideal footwear for falls prevention; (4) lifestyle factors such as incidental and formal exercise approaches that are routine and acceptable, diet, and sunlight exposure; (5) health services and systems, and patient expectations of specific health practitioners; (6) differing understanding of prevention and active engagement in prevention and intervention approaches; and (7) differences in concern about falls influencing behaviours $[23,61,63,64]$.

Given the factors outlined above, direct translation of interventions from non-Asian studies to Asian countries may warrant careful consideration. There are a number of implications for researchers, practitioners, policy and planning personnel, and research funders who may be involved in future falls prevention research in Asia. Firstly, in the area where the meta-analysis of Asian studies indicated effective interventions (Tai Chi and other exercise approaches), and where there is at least one effective randomized trial (home modifications, multiple and multifactorial interventions), that these interventions could be considered for broader implementation into practice. Two other interventions were shown to be effective in special populations: vitamin $\mathrm{K} 2$, vitamin D2 and calcium supplementation for women with probable Alzheimer's disease; and a multifactorial intervention for post hip fracture surgery patients. Even in these areas where there is some evidence of effectiveness, there is a need for research with larger samples (as the majority in this review were small samples, which limits the rigor and confidence in study findings). Furthermore, in areas like exercise, the different exercise intervention types (other than Tai Chi) have been grouped together in this review (because of the small number of studies), so there remains scope for further research exploring other exercise modalities, particularly those that may be most acceptable to Asian populations.

Secondly, for other areas of practice where there is research evidence of effective interventions in non-Asian 
populations but not in Asian populations, a number of options are available. For those interventions where research has been conducted in Asian countries but was not shown to be effective (eg knowledge/education interventions, and psychological interventions) there is a clear need to review these unsuccessful methodologies in the context of local factors that may influence their uptake and effectiveness. For example, critical elements to a successful falls prevention education program include that participants understand that falls are preventable, and that changes in behaviour, even at later ages, can still improve risk of future falls. However, research indicates that in some Asian cultures (eg China), fatalistic beliefs about falls is a major barrier that would need to be overcome [22]. Researchers and practitioners need to undertake research to improve understanding of these beliefs, and strategies that may influence these beliefs. Recent research in Australia has shown a World Café approach to be valuable in informing understanding of factors that would influence uptake and sustained engagement in falls prevention among older people, [65] and these factors have been introduced into a peer education program that was effective in increasing intention to engage in falls prevention activities [66]. Innovative, culturally relevant approaches to understanding these factors in Asian countries, and approaches to achieve sustained behaviour change (which may include these or other culturally relevant approaches) are foundational to achieving improved falls related outcomes. In using results from these type of local studies, practitioners can implement these interventions in a blended manner that retains as much of the original intervention approach as possible, but with local tailoring. For other types of falls prevention interventions that have not been investigated in Asian samples (eg medication review/reduction) there would be merit in establishing local factors as described above, prior to researchers in Asia seeking funding for local research to evaluate a culturally tailored intervention's effectiveness in reducing falls or falls injuries. In the context of medication review, inclusion of traditional and herbal medicines (which are widely used in some Asian countries), as well as interactions with pharmacy medicines would be important considerations. There may be value for this research to be collaborative with researchers who have implemented effective randomized controlled trials in non-Asian countries.

Finally, there may be other novel, locally relevant intervention types that have not been researched, that may warrant funding being sought for these interventions to be evaluated in an Asian context.

For the purposes of this research we have grouped countries under the broad umbrella classification of "Asia", however it is important to recognize that there is considerable diversity between some of the countries in
Asia, and even within some countries (for example, in Malaysia where there are three significant ethnic populations), or between urban and rural populations, where socioeconomic and other differences in some Asian countries can be stark. Specific understanding of local, cultural and societal factors that may influence acceptability of interventions need to be considered when implementing falls prevention interventions in Asian countries.

Although we have identified only a relatively small number of randomized controlled trials investigating falls prevention approaches in Asian countries, there appears to be a steady growth in the number, size and quality of the studies published more recently (since the 2012 Cochrane review) [3]. There are also two protocol papers published for studies that are underway in Asia, including a multifactorial intervention for older people with recent history of falls or injuries from falls in Malaysia $(n=300)$, [67] and an evaluation of the use of exercise using Nintendo ${ }^{\circ}$ Wii in Singapore $(n=80)$, [68] that will add to the small but growing volume of falls prevention research in this region. Of note, a number of the more recent studies are substantially larger than the median sample size of 150 for studies from Asian countries reported in the Cochrane review, which strengthens confidence in the study findings. However, the majority focussed specifically on exercise interventions only, and all were from more well developed countries in Asia.

There were several limitations to this review. A moderate limitation given the focus of this review on studies conducted in Asian countries is that only studies published in English have been included, therefore some Asian studies published in languages other than English may not have been identified. Another limitation is the varying falls outcomes published, which did not allow for additional meta-analyses to be undertaken. It would be beneficial for future falls prevention studies to include standardized outcomes to make direct comparisons and meta-analyses possible.

\section{Conclusions}

In summary, this focussed review of community setting falls prevention randomized controlled trials conducted in Asia found limited evidence of a small number of effective intervention types, relative to the strong evidence across a range of intervention types from non-Asian countries. Exercise approaches have had the strongest level of investigation, and several exercise approaches, in particular Tai Chi, have been shown to be effective. There is a need for substantial investment in large, adequately powered randomized controlled trials evaluating falls prevention interventions across Asia, in particular that incorporate tailoring of intervention approaches to the local Asian context, in order to reduce the projected escalating impact of falls in this rapidly aging part of the world. 


\section{Additional file}

Additional file 1: PRISMA checklist. (DOC $64 \mathrm{~kb}$ )

\section{Abbreviations}

CBT: Cognitive Behavioral Therapy; OR: Odds Ratio; ProFaNE: Prevention of Falls Network Europe; RCT: randomised controlled trial

\section{Acknowledgements}

The research team acknowledge the contribution of Eileen Boyle (EJB) for assistance with reviewing some of the post 2012 falls preventions studies in Asia.

\section{Funding}

There was no funding associated with this publication.

\section{Availability of data and materials}

The main study data is the data extraction materials and quality ratings of included papers, most of which are included in the manuscript tables. Any other supporting data relating to this review is available from the authors.

\section{Authors' contributions}

$\mathrm{KH}, \mathrm{PS}$, and $\mathrm{EB}$ were involved in conception and design of the study; $\mathrm{KH}, \mathrm{EB}$, and $\mathrm{KF}$ were involved in acquisition and analysis of data; $\mathrm{KH}, \mathrm{PS}, \mathrm{SL}, \mathrm{WT}, \mathrm{AA}$, $\mathrm{AH}, \mathrm{KF}$, EB were involved in interpretation of data. $\mathrm{KH}$, EB and $\mathrm{KF}$ drafted the manuscript, and $\mathrm{KH}, \mathrm{PS}, \mathrm{SL}, \mathrm{WT}, \mathrm{AA}, \mathrm{AH}, \mathrm{KF}, \mathrm{EB}$ were involved in revising the manuscript critically for important intellectual content. All authors $(\mathrm{KH}, \mathrm{PS}, \mathrm{SL}$, $\mathrm{WT}, \mathrm{AA}, \mathrm{AH}, \mathrm{KF}, \mathrm{EB}$ ) have provided final approval of the version of the manuscript submitted for publication, and all authors (KH, PS, SL, WT, AA, AH, $\mathrm{KF}, \mathrm{EB})$ agree to be accountable for all aspects of the work.

\section{Ethics approval and consent to participate}

Not applicable - This publication is a systematic review of already published papers. No ethics approvals or consent to participate were required for this work

\section{Consent for publication}

Not applicable - No details, images, or videos relating to an individual person have been included in this manuscript, therefore no consent to publish is required.

\section{Competing interests}

The authors declare that they have no competing interests.

\section{Publisher's Note}

Springer Nature remains neutral with regard to jurisdictional claims in published maps and institutional affiliations.

\section{Author details}

${ }^{1}$ School of Physiotherapy and Exercise Science, Curtin University, GPO Box U1987, Perth, WA 6845, Australia. ${ }^{2}$ Physical Therapy Department, Faculty of Allied Health Sciences, Thammasat University, Bangkok, Pathumthani, Thailand. ${ }^{3}$ Department of Physical Therapy, National Cheng Kung University, Tainan, Taiwan. ${ }^{4}$ Department of Rehabilitation Sciences, Hong Kong Polytechnic University, Hung Hom, Hong Kong. ${ }^{5}$ Malaysian Research Institute on Ageing, Universiti Putra Malaysia, Serdang, Selangor Darul Ehsan, Malaysia.

Received: 14 August 2017 Accepted: 12 December 2017

Published online: 05 January 2018

\section{References}

1. Global Burden of Disease Study 2013 Collaborators: Global, regional, and national incidence, prevalence, and years lived with disability for 301 acute and chronic diseases and injuries in 188 countries, 1990-2013: a systematic analysis for the global burden of disease study 2013. Lancet.2015, 386(9995): 743-800.

2. World Health Organisation: WHO Global report on falls prevention in older age. In.: World Health Organisation, ISBN 978924156353 6; 2007.

3. Gillespie LD, Robertson MC, Gillespie WJ, Sherrington C, Gates S, Clemson LM, Lamb SE. Interventions for preventing falls in older people living in the community. Cochrane Database Syst Rev. 2012;9:CD007146.
4. Pointer S: Trends in hospitalised injury, Australia: 1999-00 to 2012-13. In Canberra: Australian Institute of Health and Welfare (AlHW) 2015.

5. Hill $A D$, Pinto $R$, Nathens $A B$, Fowler RA. Age-related trends in severe injury hospitalization in Canada. J Trauma Acute Care Surg. 2014;77(4):608-13.

6. Orces $\mathrm{CH}$, Alamgir $\mathrm{H}$. Trends in fall-related injuries among older adults treated in emergency departments in the USA. Inj Prev. 2014;20(6):421-3.

7. Ezeh AC, Bongaarts J, Mberu B. Global population trends and policy options. Lancet. 2012;380(9837):142-8.

8. Population Reference Bureau: World population data sheet 2014. In Washington DC, USA - website: http://www.prb.org/publications/ datasheets/2014/2014-world-population-data-sheet/data-sheet.aspx; 2016.

9. Tey NP, Siraj SB, Kamaruzzaman SB, Chin AV, Tan MP, Sinnappan GS, Muller AM. Aging in multi-ethnic Malaysia. Gerontologist. 2015;

10. Chongsuvivatwong V, Phua KH, Yap MT, Pocock NS, Hashim JH, Chhem R, Wilopo SA, Lopez AD. Health and health-care systems in southeast Asia: diversity and transitions. Lancet. 2011;377(9763):429-37.

11. United Nations: World population prospects: the 2015 revision, Key Findings and Advance Tables.. In.: United Nations, Department of Economic and Social Affairs, Population Division 2015.

12. Dolinis J, Harrison J, Andrews G. Factors associated with falling in older Adelaide residents. Aust N Z J Public Health. 1997:21:462-8.

13. Campbell J, Borrie M, Spears G. Risk factors for falls in a community-based prospective study of people 70 years and older. J Gerontol. 1989:44:M112-7.

14. Koski K, Luukinen H, Laippala P, Kivela SL. Risk factors for major injurious falls among the home-dwelling elderly by functional abilities. A prospective population-based study. Gerontology. 1998;44(4):232-8.

15. Kwan MM, Close JC, Wong AK, Lord SR. Falls incidence, risk factors, and consequences in Chinese older people: a systematic review. J Am Geriatr Soc. 2011;59(3):536-43.

16. Chu LW, Chi I, Chiu AY. Incidence and predictors of falls in the chinese elderly. Ann Acad Med Singap. 2005;34(1):60-72

17. Shi J, Zhou BY, Tao YK, Yu PL, Zhang CF, Qin ZH, Sun ZQ. Incidence and associated factors for single and recurrent falls among the elderly in an urban community of Beijing. Biomed Environ Sci. 2014;27(12):939-49.

18. Han BH, Ferris $R$, Blaum C. Exploring ethnic and racial differences in falls among older adults. J Community Health. 2014:39(6):1241-7.

19. Jagnoor J, Keay L, Ivers R. A slip and a trip? Falls in older people in Asia. Injury. 2013;44(6):701-2.

20. Karuncharernpanit S, Hendricks J, Toye C. Perceptions of exercise for older people living with dementia in Bangkok, Thailand: an exploratory qualitative study. Int J Older People Nursing. 2016;11(3):166-75.

21. Jagnoor J, Keay L, Jaswal N, Kaur M, Ivers R. A qualitative study on the perceptions of preventing falls as a health priority among older people in northern India. Inj Prev. 2014;20(1):29-34.

22. Horton K, Dickinson A. The role of culture and diversity in the prevention of falls among older Chinese people. Can J Aging. 2011;30(1):57-66.

23. Romli MH, Tan MP, Mackenzie L, Lovarini M, Suttanon P, Clemson L. Falls amongst older people in Southeast Asia: a scoping review. Public Health. 2017:145:96-112.

24. Liberati A, Altman DG, Tetzlaff J, Mulrow C, Gotzsche PC, loannidis JP, Clarke M, Devereaux PJ, Kleijnen J, Moher D. The PRISMA statement for reporting systematic reviews and meta-analyses of studies that evaluate healthcare interventions: explanation and elaboration. BMJ. 2009:339:b2700.

25. Lamb SE, Becker C, Gillespie LD, Smith JL, Finnegan S, Potter R, Pfeiffer K. Reporting of complex interventions in clinical trials: development of a taxonomy to classify and describe fall-prevention interventions. Trials. 2011;12:125.

26. Higgins JP, Altman DG, Gotzsche PC, Juni P, Moher D, Oxman AD, Savovic J, Schulz KF, Weeks L, Sterne JA. The Cochrane Collaboration's tool for assessing risk of bias in randomised trials. BMJ. 2011;343:d5928.

27. Higgins J, Green S. Cochrane handbook for systematic reviews of interventions5. 1.0; 2011 In; 2011. Available from: http://training.cochrane.org/handbook.

28. Iwamoto J, Suzuki H, Tanaka K, Kumakubo T, Hirabayashi H, Miyazaki Y, Sato $Y$, Takeda T, Matsumoto $H$. Preventative effect of exercise against falls in the elderly: a randomized controlled trial. Osteoporos Int. 2009;20(7):1233-40.

29. Kamide N, Shiba Y, Shibata H. Effects on balance, falls, and bone mineral density of a home-based exercise program without home visits in communitydwelling elderly women: a randomized controlled trial. J Physiol Anthropol. 2009;28(3):115-22.

30. Sato Y, Kanoko T, Satoh K, Iwamoto J. Menatetrenone and vitamin D2 with calcium supplements prevent nonvertebral fracture in elderly women with Alzheimer's disease. Bone. 2005;36(1):61-8. 
31. Suzuki T, Kim H, Yoshida H, Ishizaki T. Randomized controlled trial of exercise intervention for the prevention of falls in community-dwelling elderly Japanese women. J Bone Miner Metab. 2004;22(6):602-11.

32. Shigematsu R, Okura T, Nakagaichi M, Tanaka K, Sakai T, Kitazumi S, Rantanen T. Square-stepping exercise and fall risk factors in older adults: a single-blind, randomized controlled trial. J Gerontol A Biol Sci Med Sci. 2008;63(1):76-82.

33. Yamada M, Tanaka B, Nagai K, Aoyama T, Ichihashi N. Trail-walking exercise and fall risk factors in community-dwelling older adults: preliminary results of a randomized controlled trial. J Am Geriatr Soc. 2010;58(10):1946-51.

34. Huang HC, Liu CY, Huang YT, Kernohan WG. Community-based interventions to reduce falls among older adults in Taiwan - long time follow-up randomised controlled study. J Clin Nurs. 2010;19(7-8):959-68.

35. Huang TT, Acton GJ. Effectiveness of home visit falls prevention strategy for Taiwanese community-dwelling elders: randomized trial. Public Health Nurs. 2004:21(3):247-56.

36. Huang TT, Liang SH. A randomized clinical trial of the effectiveness of a discharge planning intervention in hospitalized elders with hip fracture due to falling. J Clin Nurs. 2005;14(10):1193-201.

37. Huang TT, Yang LH, Liu CY. Reducing the fear of falling among communitydwelling elderly adults through cognitive-behavioural strategies and intense tai chi exercise: a randomized controlled trial. J Adv Nurs. 2011;67(5):961-71.

38. Lin MR, Wolf SL, Hwang HF, Gong SY, Chen CY. A randomized, controlled trial of fall prevention programs and quality of life in older fallers. J Am Geriatr Soc. 2007:55(4):499-506.

39. Shyu YI, Liang J, CC W, JY S, Cheng HS, Chou SW, Chen MC, Yang CT, Tseng MY. Two-year effects of interdisciplinary intervention for hip fracture in older Taiwanese. J Am Geriatr Soc. 2010;58(6):1081-9.

40. Jitapunkul S. A randomised controlled trial of regular surveillance in Thai elderly using a simple questionnaire administered by non-professional personnel. J Med Assoc Thail. 1998;81(5):352-6.

41. Assantachai P, Chatthanawaree W, Thamlikitkul V, Praditsuwan R, Pisalsarakij D. Strategy to prevent falls in the Thai elderly: a controlled study integrated health research program for the Thai elderly. J Med Assoc Thail. 2002;85(2):215-22.

42. Woo J, Hong A, Lau E, Lynn H. A randomised controlled trial of tai chi and resistance exercise on bone health, muscle strength and balance in community-living elderly people. Age Ageing. 2007;36(3):262-8.

43. Yamada M, Aoyama T Fau - Arai H, Arai H Fau - Nagai K, Nagai K Fau Tanaka B, Tanaka B Fau - Uemura K, Uemura K Fau - Mori S, Mori S Fau Ichihashi N, Ichihashi N: Complex obstacle negotiation exercise can prevent falls in community-dwelling elderly Japanese aged 75 years and older. Geriatrics and Gerotology International 2012, 12(3):461-467.

44. Yamada M, Higuchi T, Nishiguchi S, Yoshimura K, Kajiwara Y, Aoyama T. Multitarget stepping program in combination with a standardized multicomponent exercise program can prevent falls in community-dwelling older adults: a randomized, controlled trial. J Am Geriatr Soc. 2013;61(10):1669-75.

45. Ohtake M, Morikagi Y, Suzuki I, Kanoya Y, Sato C. Effects of exercise on the prevention of conditions leading to the need for long-term care. Aging Clin Exp Res. 2013;25(1):49-57.

46. Kim H, Yoshida H, Suzuki T. Falls and fractures in participants and excluded non-participants of a fall prevention exercise program for elderly women with a history of falls: 1-year follow-up study. Geriatr Gerontol Int. 2014; 14(2):285-92.

47. Hirase T, Inokuchi S, Matsusaka N, Okita M. Effects of a balance training program using a foam rubber pad in community-based older adults: a randomized controlled trial. J Geriatr Phys Ther. 2015;38(2):62-70.

48. Kamei T, Kajii F, Yamamoto Y, Irie Y, Kozakai R, Sugimoto T, Chigira A, Niino N. Effectiveness of a home hazard modification program for reducing falls in urban community-dwelling older adults: a randomized controlled trial. Jpn J Nurs Sci. 2015;12(3):184-97.

49. Hwang HF, Chen SJ, Lee-Hsieh J, Chien DK, Chen CY, Lin MR. Effects of home-based tai chi and Lower extremity training and self-practice on falls and functional outcomes in older fallers from the emergency department-a randomized controlled trial. J Am Geriatr Soc. 2016;64(3):518-25.

50. Lee HC, Chang KC, Tsauo JY, Hung JW, Huang YC, Lin SI. Effects of a multifactorial fall prevention program on fall incidence and physical function in community-dwelling older adults with risk of falls. Arch Phys Med Rehabil. 2013;94(4):606-15. 615 e601

51. Leung KS, Li CY, Tse YK, Choy TK, Leung PC, Hung WW, Chan SY, Leung AH, Cheung WH. Effects of 18-month low-magnitude high-frequency vibration on fall rate and fracture risks in 710 community elderly-a clusterrandomized controlled trial. Osteoporos Int. 2014;25(6):1785-95.
52. Ashari A, Hamid TA, Hussain MR, Hill KD. Effectiveness of individualized home-based exercise on turning and balance performance among adults older than 50 yrs: a randomized controlled trial. Am J Phys Med Rehabil. 2016;95(5):355-65.

53. Ng TP, Feng L, Nyunt MS, Feng L, Niti M, Tan BY, Chan G, Khoo SA, Chan SM, Yap P, et al. Nutritional, Physical, Cognitive, and Combination Interventions and Frailty Reversal Among Older Adults: A Randomized Controlled Trial. Am J Med. 2015;128(11):1225-36. e1221

54. Sherrington C, Tiedemann A, Fairhall N, Close J, Lord S. Exercise to prevent falls in older adults: an updated meta-analysis and best practice recommendations. N S W Public Health Bulletin. 2011;22(3-4):78-83.

55. Sitja-Rabert M, Martinez-Zapata MJ, Fort-Vanmeerhaeghe A, Rey-Abella F, Romero-Rodriguez D, Bonfill X. Whole body vibration for older persons: an open randomized, multicentre, parallel, clinical trial. BMC Geriatr. 2011;11:89.

56. Hill K, Choi W, Smith R, Condron J. Tai chi in Australia: an acceptable and effective approach to improve balance and mobility in older people? A pilot study. Australas J Ageing. 2005;24:9-13.

57. Li F, Harmer P, Fisher KJ, McAuley E, Chaumeton N, Eckstrom E, Wilson NL. Tai chi and fall reductions in older adults: a randomized controlled trial. J Gerontol A Biol Sci Med Sci. 2005;60(2):187-94.

58. Hill K, Choi W, Smith R, Condron J. Tai chi in Australia: acceptable and effective approach to improve balance and mobility in older people? Australas J Ageing. 2005;24(1):9-13.

59. Sherrington C, Michaleff ZA, Fairhall N, Paul SS, Tiedemann A, Whitney J, Cumming RG, Herbert RD, Close JC, Lord SR. Exercise to prevent falls in older adults: an updated systematic review and meta-analysis. [Epub ahead of print]. Br J Sports Med. 2016;

60. Nyman SR, Victor CR. Older people's participation in and engagement with falls prevention interventions in community settings: an augment to the Cochrane systematic review. Age Ageing. 2012;41(1):16-23.

61. Kalula SZ, Scott V, Dowd A, Brodrick K. Falls and fall prevention programmes in developing countries: environmental scan for the adaptation of the Canadian falls prevention curriculum for developing countries. I Saf Res. 2011;42(6):461-72.

62. Kong NH, Chow PK. Conducting randomised controlled trials across countries with disparate levels of socio-economic development: the experience of the Asia-Pacific hepatocellular carcinoma trials group. Contemp Clin Trials. 2013;36(2):682-6.

63. Dsouza S, Rajashekar B, Dsouza H, Kumar K. Falls in Indian older adults: a barrier to active ageing. Asian J Gerontol Geriatr. 2014;9(1):1-8.

64. Kwan MM, Tsang WW, Lin SI, Greenaway M, Close JC, Lord SR. Increased concern is protective for falls in Chinese older people: the chopstix fall risk study. J Gerontol A Biol Sci Med Sci. 2013;68(8):946-53.

65. Bulsara C, Khong L, Hill K, Hill A. Investigating community perspectives on falls prevention information seeking and delivery: older person perceptions regarding preferences for falls prevention education using a world cafe approach. J Community Psychol. 2016;44(7):937-44.

66. Khong LAM, Berlach RG, Hill KD, Hill AM. Can peer education improve beliefs, knowledge, motivation and intention to engage in falls prevention amongst community-dwelling older adults? European journal of ageing. 2017;14(3):243-55

67. Tan PJ, Khoo EM, Chinna K, Hill KD, Poi PJ, Tan MP. An individually-tailored multifactorial intervention program for older fallers in a middle-income developing country: Malaysian falls assessment and intervention trial (MyFAIT). BMC Geriatr. 2014;14:78.

68. Kwok BC, Mamun K, Chandran M, Wong CH. Evaluation of the Frails' fall efficacy by comparing treatments (EFFECT) on reducing fall and fear of fall in moderately frail older adults: study protocol for a randomised control trial. Trials. 2011;12:155.

69. Tennstedt S, Howland J, Lachman M, Peterson E, Kasten L, Jette A. A randomized, controlled trial of a group intervention to reduce fear of falling and associated activity restriction in older adults. J Gerontol B Psychol Sci Soc Sci. 1998;53(6):P384-92. 